\title{
10 Ausblick und Aufgaben für die Zukunft
}

Aus der Sicht eines Kinder- und Jugendpsychiaters resultiert das Phänomen „Schul-Amok“ aus einer Fehlentwicklung junger Menschen, die einen extremen Weg wählen, um ihre seelischen Verletzungen zu bewältigen, indem sie ein Massaker an ihren Mitschülern und Lehrern ausüben. Bei der Analyse ihrer Biographien wird deutlich, dass sie selbst Opfer von Demütigungen und Kränkungen waren. Darum muss das Phänomen „Schul-Amok“ in einem gesellschaftlichen Zusammenhang gesehen werden. Auch in Zukunft wird es gegen Schul-Amokläufe kein einfaches Rezept geben, denn dies würde voraussetzen, wesentliche Faktoren in unserer Gesellschaft zu verändern. Es kommt jedoch darauf an, weitere Schul-Amoktaten mit ihren monströsen Folgen zu verhindern. Daran müssen sich alle relevanten Institutionen beteiligen:

Kinder- und Jugendpsychiatrie. Ihr Beitrag besteht in Diagnostik und Behandlung auffällig gewordener Kinder und Jugendlicher unter Einbeziehung von Bezugspersonen, insbesondere der Stärkung der Elternkompetenz.

Eltern. Sie müssen ihren Kindern den Weg ins Leben eröffnen, indem sie ihnen ein Urvertrauen vermitteln. Sie müssen Anzeichen für problematische Entwicklungen erkennen und Hilfsangebote rechtzeitig in Anspruch nehmen.

Schule. Sie darf sich nicht nur als Wissensvermittler verstehen, sondern sich verstärkt der Vermittlung sozialer und emotionaler Kompetenzen und insbesondere der Entwicklung von Empathie widmen.

Schulbehörde. Sie hat die Aufgabe, der Schule die Rahmenbedingungen zur Verfügung zu stellen, die notwendig sind, ihre gesellschaftsrelevanten Aufgaben zu erfüllen. 
Strafverfolger und Justizbehörden. Sie müssen sich verstärkt in Krisenteams einbringen, um rechtzeitig Bedrohungslagen zu erkennen und zu entschärfen.

Politik. Sie hat die Aufgabe, Bildungschancen zu verbessern und die Teilhabe der Jugend am gesellschaftlichen Leben zu ermöglichen.

Medien. Sie müssen sich selbst einem Pressecodex verpflichten, der auch die Opfer vor zusätzlichen Traumatisierungen schützt.

Forschung. Ihre Aufgabe wird es sein, u.a. den Zusammenhang von „Sexualität und Gewalt" besser verstehen zu lernen und darzustellen. Ein weiterer Gesichtpunkt ist, die „Theorie der strukturellen Dissoziation“ auf ihre Nützlichkeit im Hinblick auf das Phänomen „Schul-Amok“ zu prüfen und neue Behandlungskonzepte daraus abzuleiten.

Alle am Erziehungsprozess Beteiligte. Für sie muss es das Ziel sein, eine Kultur des Hinschauens, des Verständnisses und der Übernahme von Verantwortung zu entwickeln. Sie befähigen Kinder und Jugendliche, Regeln auszuhandeln und einzuhalten, Widersprüche und Andersartigkeiten zu akzeptieren und angemessene Konfliktlösungen zu entwickeln. 